\title{
छூ \\ Effects of ion bombardment on bulk GaAs photocathodes with different surface-cleavage planes
}

\author{
Wei Liu, ${ }^{1,2,3, *}$ Shukui Zhang, ${ }^{3}$ Marcy Stutzman, ${ }^{3}$ and Matt Poelker ${ }^{3, \dagger}$ \\ ${ }^{1}$ Institute of Modern Physics, Chinese Academy of Sciences, 509 Nanchang Road, Lanzhou, 730000, China \\ ${ }^{2}$ University of Chinese Academy of Sciences, 19 A Yuquan Road, Beijing 100049, China \\ ${ }^{3}$ Thomas Jefferson National Accelerator Facility, 12000 Jefferson Avenue, \\ Newport News, Virginia 23606, USA
}

(Received 22 July 2016; published 24 October 2016)

Bulk GaAs samples with different surface cleave planes were implanted with 100 and $10000 \mathrm{~V}$ hydrogen ions inside an ultrahigh vacuum test apparatus to simulate ion back-bombardment of the photocathode inside a DC high voltage photogun. The photocathode yield, or quantum efficiency, could easily be recovered following implantation with $100 \mathrm{~V}$ hydrogen ions but not for $10000 \mathrm{~V}$ ions. Moreover, the implantation damage with $10000 \mathrm{~V}$ hydrogen ions was more pronounced for GaAs photocathode samples with (100) and (111A) cleave planes, compared to the photocathode with (110) cleave plane. This result is consistent with enhanced ion channeling for the (110) cleave plane compared to the other cleave planes, with ions penetrating deeper into the photocathode material beyond the absorption depth of the laser light and beyond the region of the photocathode where the photoemitted electrons originate.

DOI: 10.1103/PhysRevAccelBeams.19.103402

\section{INTRODUCTION}

Direct current high voltage photoemission guns with GaAs photocathodes are used to generate spin-polarized electron beams for nuclear and particle physics research [1]. More recently, these photoguns have found application in material science where spin-polarized electron beams can be used for magnetization-sensitive imaging [2]. Direct current high voltage photoguns with GaAs $[3,4]$ or alkaliantimonide photocathodes $[5,6]$ are also used to produce bright electron beams for accelerator-based light sources.

Ion back-bombardment represents the dominant lifetimelimiting mechanism of modern dc high voltage photoguns $[7,8]$. Ion back-bombardment is the process whereby residual gas within the cathode/anode gap is ionized by the extracted electron beam and attracted to the negatively biased photocathode. Photogun experts speculate that ions strike the photocathode surface sputtering away the chemicals used to create a negative electron affinity (NEA) condition, or they become implanted within the photocathode material knocking out atoms from the crystal structure and altering the energy band structure, or they serve as interstitial defects that reduce the electron diffusion length. All of these possibilities are problematic because the net result is reduced photocathode yield, or quantum

\footnotetext{
weiliu1006@yahoo.com

†poelker@jlab.org
}

Published by the American Physical Society under the terms of the Creative Commons Attribution 3.0 License. Further distribution of this work must maintain attribution to the author(s) and the published article's title, journal citation, and DOI. efficiency $(\mathrm{QE})$, which in turn results in reduced operating lifetime of the photogun. Improving the vacuum within the photogun is the best way to reduce ion back-bombardment and thereby prolong the operating lifetime of the photogun, but modern dc high voltage photoguns are already operating in the $10^{-11}$ Torr pressure range, or lower, so improving the photogun vacuum further is thus very challenging.

In this study, we explored the sensitivity of bulk GaAs photocathodes with (100), (110) and (111A) surface cleave planes to ion bombardment at hydrogen-ion energies of 100 and $10000 \mathrm{~V}$. Tests were performed using an ultrahigh vacuum chamber where GaAs photocathodes were created in the usual manner, with the photocathode biased at low voltage and with the surface work function reduced by applying cesium and an oxidant $\left(\mathrm{NF}_{3}\right)$ to create a NEA condition. After verifying that each sample provided high $\mathrm{QE}$ at $532 \mathrm{~nm}$, the NEA layer was removed. Then samples were implanted with hydrogen ions from an atomic hydrogen source, originally used to clean photocathodes $[9,10]$, but used here as an ion source, The energy of the implanted ions was determined by the value of the negative bias applied to the photocathode (more details provided below). Samples were then heated and reactivated-i.e., chemicals were reapplied to the GaAs surface to recreate the NEA condition. Repeated heating and activation using different heating protocols (temperature and duration) provided a means to evaluate a sample's sensitivity to ion bombardment. A central motivation of this study was to determine the susceptibility of each cleave plane to ion damage, and to what extent ion channeling $[11,12]$ plays a role in $\mathrm{QE}$ reduction. We sought to test if enhanced ion channeling would serve to deliver ions deeper into the photocathode 


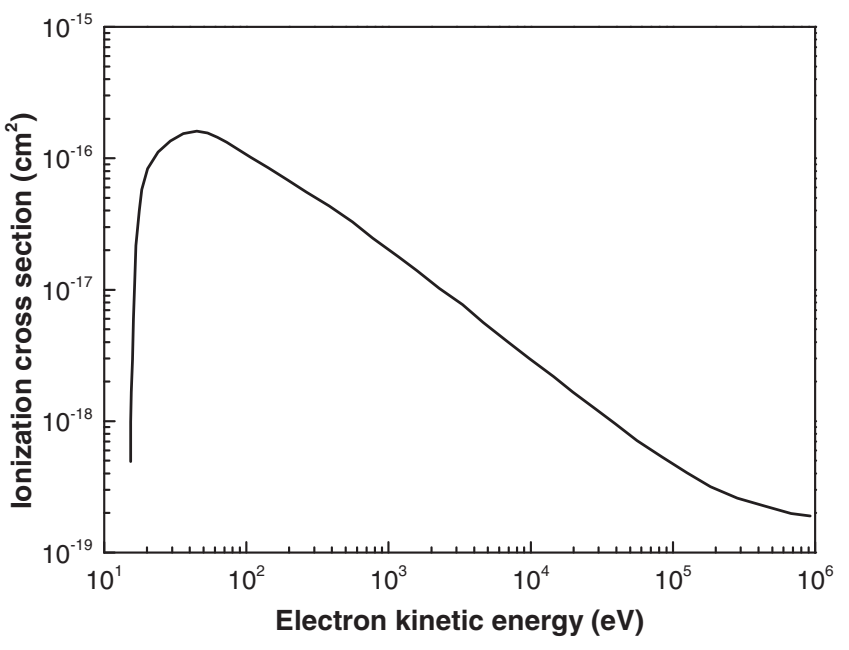

FIG. 1. Electron impact ionization cross section for hydrogen molecules-the dominant gas species inside modern dc high voltage photoguns - as a function of electron kinetic energy.

material, beyond the region near the surface of the photocathode where the photoemitted electrons originate. Enhanced ion channeling could benefit a photogun, minimizing the ill effects of ion bombardment and prolonging the photogun operating lifetime.

To understand ion back-bombardment inside a photogun, it is important to note that the energy spectrum of the extracted photoemitted electron beam within the cathode/ anode gap is broad, with electrons leaving the cathode electrode at zero velocity, and then gaining energy until reaching the anode. For a typical dc high voltage photogun, the photocathode is biased at voltages between -100 and $-350 \mathrm{kV}$. Hydrogen is the dominant gas species inside a modern photogun, and the peak ionization cross section occurs at $\sim 100 \mathrm{~V}$ and drops by more than 2 orders of magnitude at $100 \mathrm{kV}[8,13]$ (Fig. 1). So under typical photogun operation, the surface of the photocathode is bombarded by significantly more low energy ions than high energy ions. Determining the sensitivity of photocathode $\mathrm{QE}$ to ion energy represents another key motivation of this study.

Since the late 1960s [14,15], GaAs has been extensively studied for its properties as an effective photoemitter. James et al. [16], working with vacuum-cleaved samples, reported that (100) and (110) surfaces-with equal amounts of Ga and As atoms at the surface-provide similar QE as a function of the illumination wavelength (i.e., QE spectral response), whereas the (111A) surface comprised of only $\mathrm{Ga}$ atoms has the lowest QE and the (111B) surface comprised of only As atoms has the highest QE. Today it is a trivial matter to purchase high quality GaAs samples with the desired cleave plane surface. For this work, three diameter bulk GaAs wafers were purchased from a commercial vendor with (100), (110) and (111A) surface cleave planes. All samples were p-doped with $\mathrm{Zn}$ at nominal concentration $\sim 1 \times 10^{19}$ atoms $/ \mathrm{cm}^{3}$. Other characteristics are provided in Table I. No chemical preparation was performed on samples prior to installation in the vacuum test chamber. They were cleaved into $15 \times 15 \mathrm{~mm}$ squares and attached to a sample holder, described below. This work also serves to assess which cleave plane surface provides the highest $\mathrm{QE}$, using commercially available material and modern vacuum hardware and techniques.

Whereas ion implantation in the form of ion backbombardment is detrimental to the photocathode inside a dc high voltage photogun, ion implantation is beneficial and widely used in the fabrication of integrated circuits. As a result, implantation has been extensively studied [17]. The nuclei and the electrons within a crystallographic plane repel a positively charged ion like the hydrogen ions used in this experiment. As a result, positively charged particles tend to follow the direction between two neighboring crystal planes. This process-called channeling-leads to deeper penetration of the positive ions in the material. Channeling is more pronounced at higher ion energies. Figure 2 shows the relative spacing of atoms in the GaAs crystal structure for the three surface cleave planes studied, viewed at normal incidence. The red boxes in Fig. 2 denote the recurring geometric cells used for calculating the atomic number density which is a measure of the open space between atoms. The atomic number density for the (100), (110) and (111A) GaAs cleave planes were calculated to be $8 / a^{2}, 4 \sqrt{2} / a^{2}$, and $4 \sqrt{3} / a^{2}$, respectively, where $a$ is the GaAs lattice constant (5.6535 $\AA$ ). We reasoned channeling would be enhanced for the (110) cleave plane which possesses the smallest atomic number density (i.e., the most open space between crystalline layers), and that the (110) surface cleave plane would therefore be the most insensitive to ion bombardment.

TABLE I. The specifications of three bulk GaAs samples with different surface cleave planes.

\begin{tabular}{lccc}
\hline \hline & $\operatorname{GaAs}(100)$ & GaAs(110) & GaAs(111A) \\
\hline Dopant & GaAs-Zn & GaAs-Zn & GaAs-Zn \\
Orientation & $(100) \pm 0.5^{\circ}$ & $(110) \pm 0.5^{\circ}$ & $(111 A) \pm 0.5^{\circ}$ \\
Carrier concentration (a./c.c) & $1.0-1.3 \times 10^{19}$ & $1.3-1.4 \times 10^{19}$ & $1.1-1.14 \times 10^{19}$ \\
Resistivity $($ ohm. cm) & $6.6-7.7 \times 10^{-3}$ & $6.1-6.6 \times 10^{-3}$ & $7.17-7.38 \times 10^{-3}$ \\
Mobility $\left(\mathrm{cm}^{2} /\right.$ v.s. $)$ & $74-80$ & $71-74$ & $77-78$ \\
Thickness $(\mu \mathrm{m})$ & $600-650$ & $475-525$ & $500-550$ \\
\hline \hline
\end{tabular}



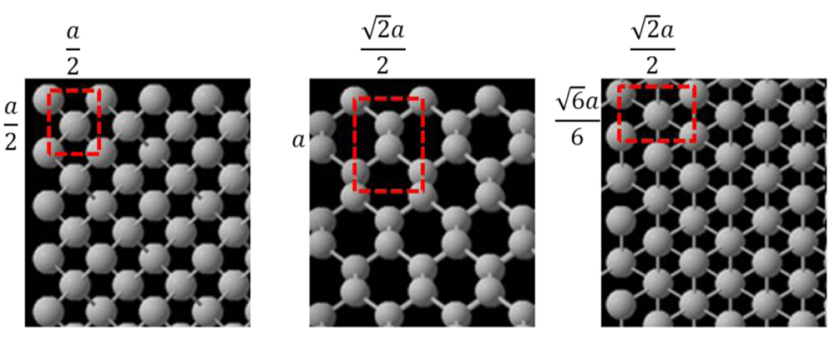

FIG. 2. Schematic representations of the GaAs crystal for three surface cleave planes, looking normal to the surface-from left to right, (100), (110) and (111A). The red boxes denote the recurring geometric cells used for calculating the atomic number density for each cleave plane and $a$ is the GaAs lattice constant.

Although the software program SRIM (stopping range of ions in matter) [18] does not take into account the crystal orientation of the GaAs photocathode, and thus does not describe channeling, the program was used to estimate the penetration depth of the hydrogen ions into the material and the number of vacancies created within the material when ions collide with gallium and arsenic atoms and knock them out of their positions within the crystal lattice. In the
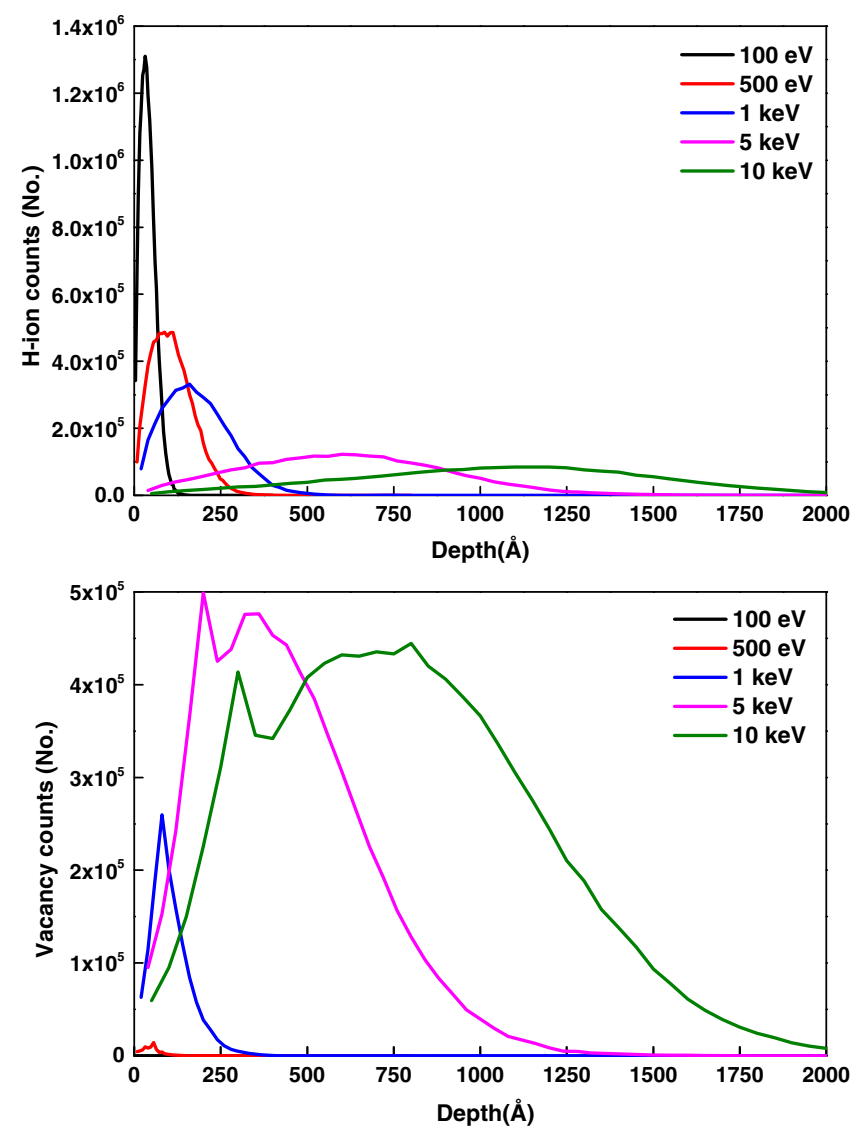

FIG. 3. (top) Hydrogen ion distribution and (bottom) vacancy distribution within GaAs for different hydrogen ion energies. All simulations performed using the same hydrogen ion dose of $8.1 \times 10^{5} \mathrm{nA}$ s. Plots were obtained using SRIM [18]. simulation, the hydrogen ions were injected into GaAs at normal incidence and the ion injection energy was varied from 100 to $10000 \mathrm{~V}$ while keeping the ion dose constant for each simulation. The stopping depth of implanted hydrogen ions and the distribution of knock-out vacancies for different ion energies are shown in Fig. 3. Unsurprisingly, higher energy ions penetrate deeper into the material and do more damage to the crystal structure compared to lower energy ions. The penetration depth (defined as the depth at which the dose falls to $1 / e$ of its original value) is $69 \AA$ and $1690 \AA$ for $100 \mathrm{~V}$ and $10000 \mathrm{~V}$ hydrogen ions, respectively, while gallium and arsenic atoms begin to be knocked out from their lattice positions at ion energies greater than $\sim 500 \mathrm{~V}$. Laser light at $532 \mathrm{~nm}$ will be absorbed within $1300-1480 \AA$ of the surface [19-21] and we note the diffusion length of electrons in GaAs is of the order $1000 \AA[22,23]$. Because these dimensions are comparable to the distance scales described in Fig. 3 for implantation and knock-out vacancies, we hoped that our QE measurements would be sensitive to different ill effects that might result from low and high energy ions at 100 and $10000 \mathrm{~V}$.

\section{APPARATUS}

The experiment followed a sequence of steps. A photocathode sample was attached to a long tubular sample holder and installed in the vacuum test chamber which was then baked to achieve ultrahigh vacuum. Afterwards, the sample was heated to $550^{\circ} \mathrm{C}$ for $1 \mathrm{~h}$ and allowed to cool. A photocathode was manufactured by applying cesium and $\mathrm{NF}_{3}$ to the surface using a standard yo-yo activation protocol and afterwards the QE was mapped using a low power green laser beam that could be scanned across the photocathode surface. The sample was then biased at either 100 or $10000 \mathrm{~V}$ and exposed to hydrogen ions of a prescribed dose. Finally, the sample was heated and reactivated multiple times to observe QE evolution. Additional specific details are provided below.

Square-shaped photocathode samples were cleaved to appropriate dimension $(15 \times 15 \mathrm{~mm})$ using a diamond tipped scribe and secured to a molybdenum disk at the end of a tubular holder using indium foil and a tantalum cap with $13 \mathrm{~mm}$ diameter cutout in the center. During installation of the photocathode sample, care was taken to limit the amount of water vapor introduced into the apparatus (Fig. 4) by applying a vigorous flow of clean dry nitrogen through the vacuum pump cart. After tightening the sample holder Conflat flange, the flow of nitrogen through the apparatus was halted and the chamber was evacuated using a turbo pump. When pressure within the chamber reached $\sim 10^{-6}$ Torr, electrical current was applied to the substrate of the nonevaporable getter pumps to liberate adsorbed gas, and the ion pump was energized. A prebake leak check was performed using a residual gas analyzer and oven panels were erected around the chamber which was then baked at 


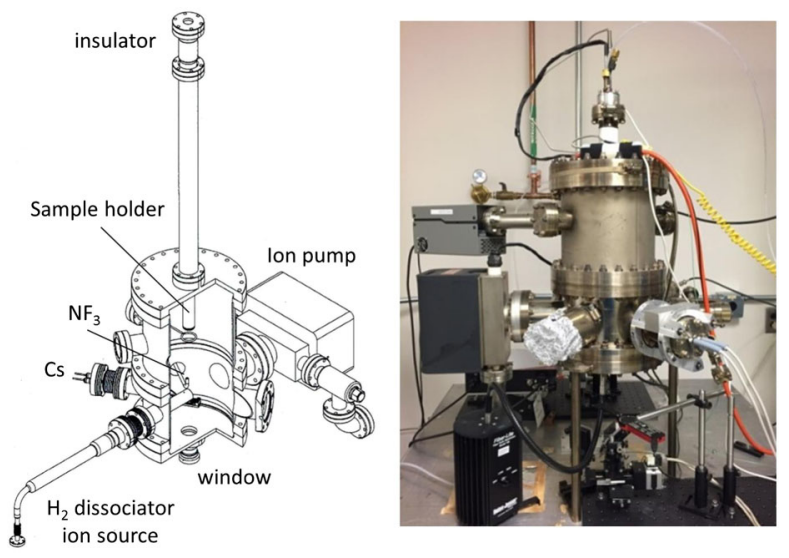

FIG. 4. Schematic representation of the ultrahigh vacuum test apparatus (left) and a photograph of the apparatus (right).

$200^{\circ} \mathrm{C}$ for $36 \mathrm{~h}$. Following the bakeout, the pressure within the chamber reached $10^{-11}$ Torr.

A heat cycle always immediately preceded photocathode activation. The photocathode was heated to $550^{\circ} \mathrm{C}$ for $1 \mathrm{~h}$ before the first activation, and at different temperatures and durations for activations following ion implantation. Heat liberates loosely bound gas at the photocathode surface and it liberates hydrogen atoms and molecules that penetrate the photocathode surface during the ion implantation phase of the experiment. After the sample was cooled to room temperature, cesium and $\mathrm{NF}_{3}$ were applied to reduce the surface work function. The sample was biased at $-250 \mathrm{~V}$ and illuminated with a white light source. A yo-yo activation protocol was employed-cesium was applied until photocurrent reached a peak and then decayed to approximately half the maximum value. At this point, the cesium source was turned OFF and a leak valve was opened to introduce NF3 into the apparatus. Photocurrent was observed to increase to a new peak value. The $\mathrm{NF}_{3}$ leak valve was then closed and additional cesium was added, which caused the photocurrent to decrease. When photocurrent decreased to approximately half the new maximum value, the cesium source was deenergized and more $\mathrm{NF}_{3}$ applied, each time providing more photocurrent than the previous cycle. This so-called yo-yo procedure was repeated approximately 12 times until the new peak current was within $\sim 3 \%$ of the previous cycle maximum. With photocathode activation complete, the photocathode QE was mapped by a scanning focused laser beam at $532 \mathrm{~nm}$. Because vacuum conditions inside the apparatus were very good, the lifetime of the photocathode was very long, and photocathode QE remained stable during the QE x-y scan and for many hours afterwards.

The hydrogen ion source is based on work in Ref. [24]. Commercial research-grade molecular hydrogen flows through a leak valve into a Pyrex glass dissociator $(2.5 \mathrm{~cm}$ diameter). The molecular hydrogen was dissociated using an rf-inductive discharge created by a 12 turn coil $3.5 \mathrm{~cm}$ in
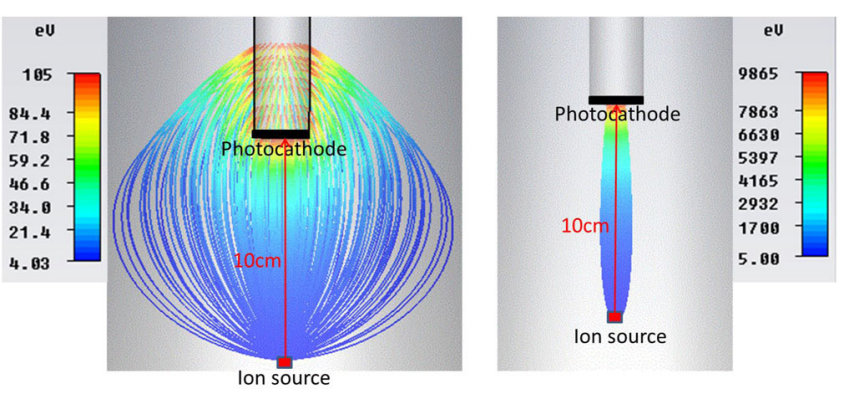

FIG. 5. Ion trajectory from the hydrogen ion source to the photocathode sample when the photocathode sample is biased at $100 \mathrm{~V}$ (left) and $10000 \mathrm{~V}$ (right). Ions exit the dissociator glassware from a $1 \mathrm{~mm}$ diameter hole located approximately $10 \mathrm{~cm}$ beneath the photocathode sample.

diameter, which was part of an inductor-capacitor (LC) tuned circuit. The LC circuit resonated at $\sim 100 \mathrm{MHz}$ and the atomic fraction was maximized when the pressure inside the dissociator was 15 mTorr and the absorbed rf power exceeded $50 \mathrm{~W}$. Gas exits the dissociator through a $1 \mathrm{~mm}$ diameter hole toward the photocathode sample approximately $10 \mathrm{~cm}$ away. A portion of the gas leaving the dissociator is ionized, and these ions were directed at the sample by applying a negative bias to the sample holder which was electrically isolated from the grounded apparatus using a commercial ceramic break. During implantation, a valve was opened to a baked beam line leading to a turbomolecular pump. This pumping scheme serves to maintain pressure near the sample $\sim 1 \times 10^{-5}$ Torr to provide a mean-free path for hydrogen of over $1 \mathrm{~m}$.

As previously mentioned, two different hydrogen ion implantation energies were studied, 100 and $10000 \mathrm{~V}$. An ammeter in series with the photocathode bias-voltage power supply was used to continuously monitor the ion current at the sample, and the time integrated current represented the applied ion dose. A LABVIEW program was used to monitor and integrate the real-time dose and the implantation was stopped when the dose reached $8.1 \times 10^{5} \mathrm{nAs}$, corresponding to a typical ion current of $900 \mathrm{nA}$ and for an implantation duration of $15 \mathrm{~min}$.

Hydrogen ions leave the source at relatively low energy $(\sim \mathrm{eV})$ and travel in different directions. With no bias voltage applied to the sample, few ions reach the photocathode, as evidenced by the ammeter which registers low current ( $<10 \%$ of current with bias voltage). But when the sample is biased at negative voltage, the ions are attracted to the sample (and the sample holder). The software program CST Studio Suite [25] was used to evaluate the electrostatic conditions of the apparatus and to estimate ion trajectories for the two bias voltages (see Fig. 5). Although the number of ions emitted from the source and the duration of exposure were nominally identical for both energies, the dose at $100 \mathrm{~V}$ was approximately a factor of 2 smaller compared to $10000 \mathrm{~V}$ trials because roughly half the ions struck the sample holder due to weaker 
electrostatic focusing. The applied dose per unit area was an additional factor of 10 smaller for the $100 \mathrm{~V}$ trials based on simple geometric considerations, namely $100 \mathrm{~V}$ ions illuminated the entire $13 \mathrm{~mm}$ diameter sample, whereas $10000 \mathrm{~V}$ ions were focused at the sample within a region $\sim 4 \mathrm{~mm}$ diameter. There was no accounting for possible secondary electron emission from the sample at either energy.

\section{RESULTS}

Prior to implantation, each photocathode sample was heated, cooled and activated. The QE was then measured as a function of laser wavelength and the results are presented in Fig. 6. Quantum efficiency was calculated using the expression $Q E=124 I /(P \lambda)$ [26], where I is the measured photocurrent in $\mathrm{A}, \mathrm{P}$ is the laser power in $\mathrm{mW}$, and is the wavelength of the laser light in $\mathrm{nm}$. The cleave planes (100) and (110) provided the highest QE, approximately $21 \%$ at the laser wavelength $532 \mathrm{~nm}$. Cleave plane sample (111A) provided the lowest QE, approximately $19 \%$ at $532 \mathrm{~nm}$. Although these values are lower than reported in Ref. [16], the relative trend between samples is consistent (i.e., $\left.Q E_{100}>Q E_{110}>Q E_{111 A}\right)$.

After determining that each sample provided high $\mathrm{QE}$, samples were heated to $250^{\circ} \mathrm{C}$ for $20 \mathrm{~min}$ to remove the activation layer of $\mathrm{Cs}$ and $\mathrm{NF}_{3}$. Samples were then cooled to room temperature and implanted with hydrogen ions at the chosen energy and for the prescribed dose. The vacuum was allowed to recover overnight. Photocathode samples were then repeatedly heated and activated following different heating protocols (temperature and duration). Following each activation, the photocathode QE was measured at $532 \mathrm{~nm}$ and compared to the preimplantation value. In this manner, the sample's sensitivity to ion

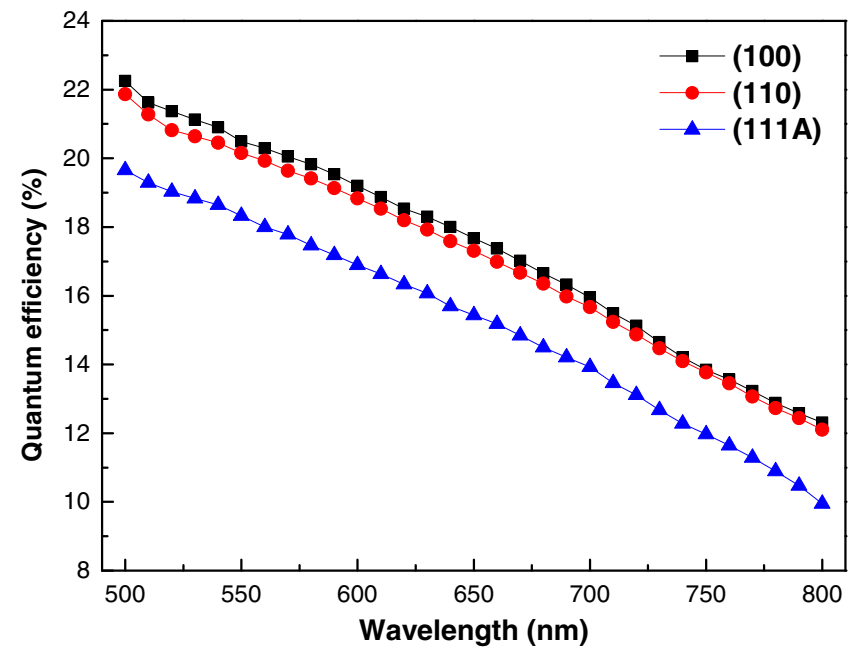

FIG. 6. Photocathode QE versus laser wavelength for commercial bulk GaAs samples with different surface cleave planes, prior to ion implantation.
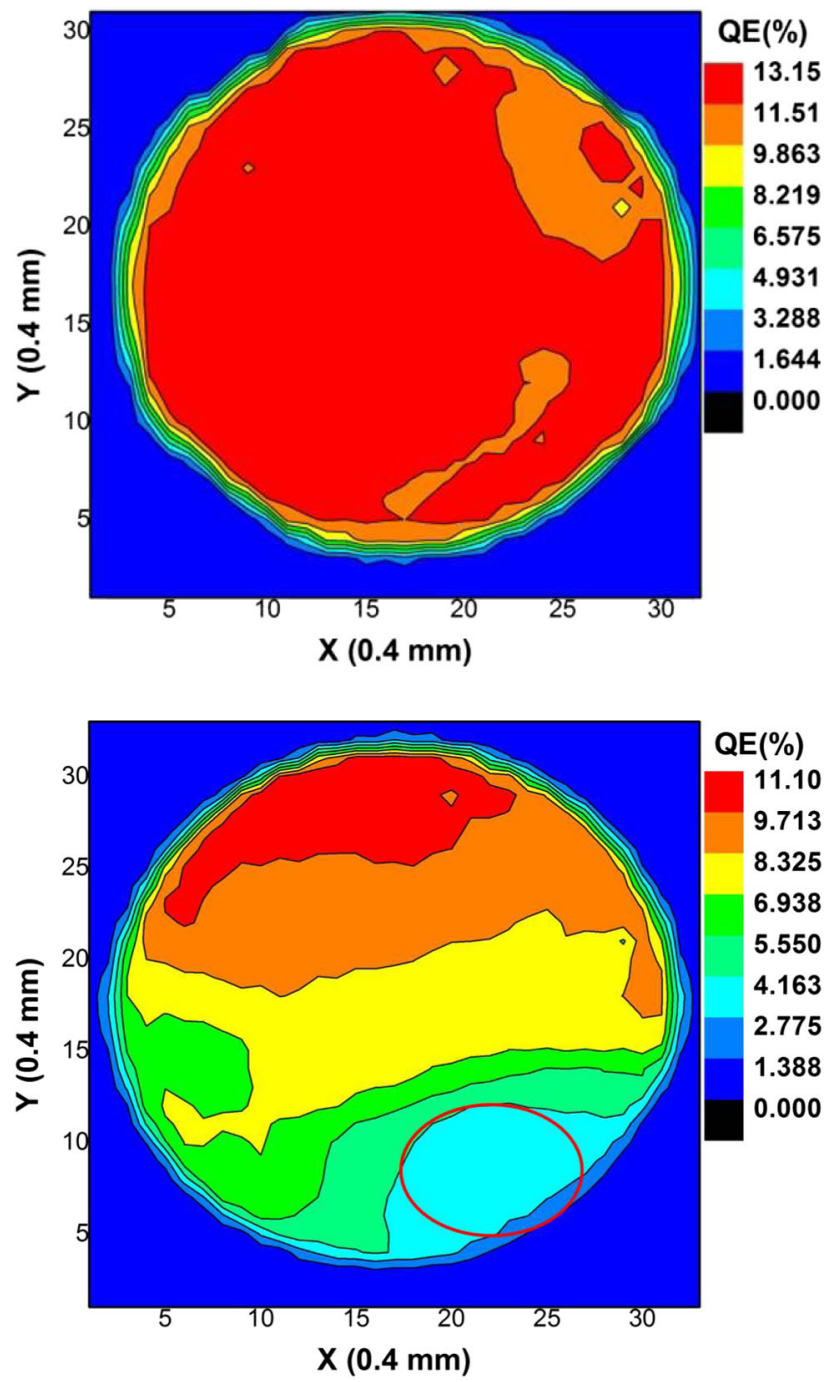

FIG. 7. Typical QE maps of the photocathode obtained by scanning a focused low power green laser beam across the photocathode following activation. These maps show the bulk GaAs (111A) sample. Top: After the third activation following implantation with $100 \mathrm{~V}$ hydrogen ions. Bottom: After the third activation following implantation with $10000 \mathrm{~V}$ hydrogen ions. The two-dimensional plots help to illustrate ion implantation uniformity: $100 \mathrm{~V}$ hydrogen ions were uniformly distributed across the entire sample but $10000 \mathrm{~V}$ hydrogen ions were focused to a region denoted by the red circle, consistent with the particle tracking code simulations.

damage was evaluated. Figure 7 shows typical QE maps for samples implanted with 100 and $10000 \mathrm{~V}$ hydrogen ions (the caption provides specific details of the cleave plane surface and heating protocol). For $100 \mathrm{~V}$ ions, QE degradation was uniformly distributed across the sample. But for $10000 \mathrm{~V}$ hydrogen ions, the QE degradation was localized to a specific region of the sample, consistent with the electrostatic simulation that predicted a focused ion beam. Because the ion damage was displaced from the center of the photocathode, we assume the output hole of 


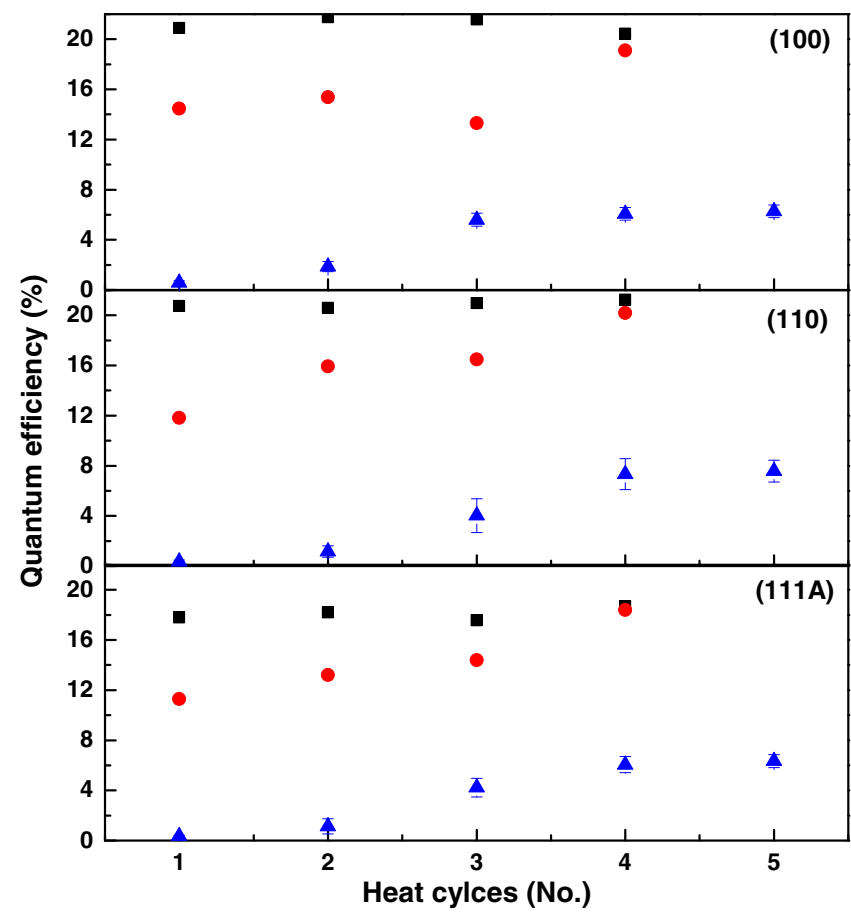

FIG. 8. QE versus heat cycles for bulk GaAs with three different surface cleave planes. Black square: no implantation; red circle: implantation with $100 \mathrm{~V}$ hydrogen ions; blue triangle: implantation with $10000 \mathrm{~V}$ hydrogen ions. Errors for no implantation and $100 \mathrm{~V}$ results are small because QE was uniform across sample; errors for $10000 \mathrm{~V}$ results are large because ion damage was limited to small region of photocathode. Heat cycle numbers: $1: 250^{\circ} \mathrm{C}$ for $20 \mathrm{~min} ; 2: 370^{\circ} \mathrm{C}$ for $30 \mathrm{~min}$; 3: $490^{\circ} \mathrm{C}$ for $1 \mathrm{~h} ; 4$ and 5: $550^{\circ} \mathrm{C}$ for $1 \mathrm{~h}$.

the hydrogen dissociator was misaligned relative to the photocathode sample. The misalignment was along the left/ right axis of the dissociator, which could not easily be corrected with existing vacuum hardware. The damage site location remained the same throughout the experiment, for each implantation and each sample.

Figure 8 summarizes the results of the experiment, with $\mathrm{QE}$ at $532 \mathrm{~nm}$ plotted as a function of repeated activation following a variety of heating protocols described within the caption, for samples implanted with 100 and 10,000 V hydrogen ions, and for samples that were not implanted with ions. When samples were repeatedly heated and activated - but not subjected to hydrogen ion implantationthe $\mathrm{QE}$ at $532 \mathrm{~nm}$ remained constant to within $5 \%$. This speaks to the quality of the vacuum within the apparatus, which was dominated by chemically inert gas species like $\mathrm{H}_{2}$, $\mathrm{CH}_{4}, \mathrm{CO}$ and $\mathrm{CO}_{2}$. The $\mathrm{QE}$ of each sample did not degrade following repeated heating and activation, due to adverse chemical reactions that might take place under degraded vacuum conditions, especially when samples were hot.

The next observation evident in Fig. 8 is that both 100 and $10000 \mathrm{~V}$ hydrogen ions served to degrade the sample $\mathrm{QE}$, however the QE degradation associated with $10000 \mathrm{~V}$

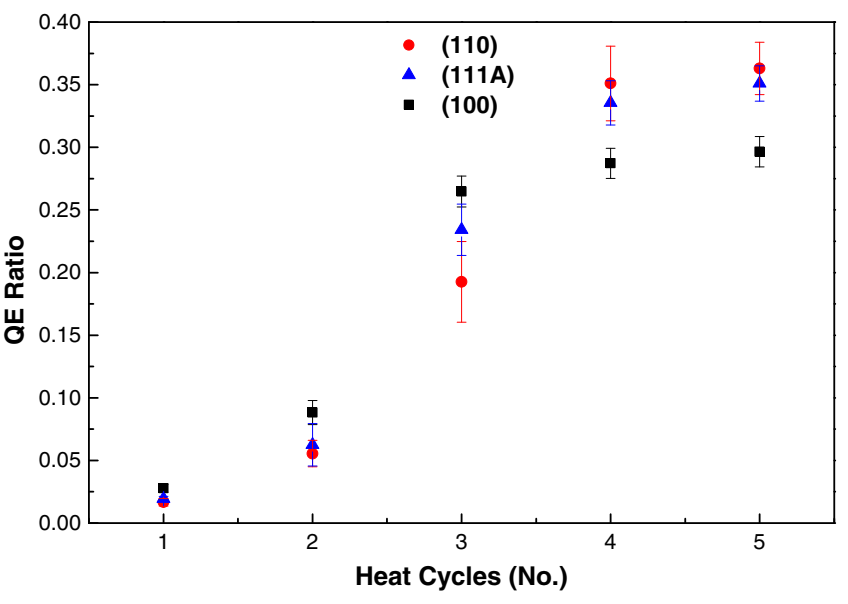

FIG. 9. QE ratio-samples implanted with $10000 \mathrm{~V}$ hydrogen ions to samples that were not exposed to hydrogen ions-as a function of heat cycle, for bulk GaAs with three different surface cleave planes. Heat cycle numbers: $1: 250^{\circ} \mathrm{C}$ for $20 \mathrm{~min}$; 2: $370^{\circ} \mathrm{C}$ for $30 \mathrm{~min} ; 3: 490^{\circ} \mathrm{C}$ for $1 \mathrm{~h} ; 4$ and 5: $550^{\circ} \mathrm{C}$ for $1 \mathrm{~h}$.

ions was significantly greater than that caused by $100 \mathrm{~V}$ ions. In general, for both ion energies, heating the sample to higher temperature and for longer time served to restore QE. For implantation with $100 \mathrm{~V}$ hydrogen ions, the QE could be completely restored by heating the sample. But for implantation with $10000 \mathrm{~V}$ hydrogen ions, only a fraction of the QE could be restored. It should be noted that data points presented in Fig. 8 were obtained by averaging the QE measurements over a specific region of the photocathode sample surface. For $100 \mathrm{~V}$ ion implantation, QE measurements were averaged over the entire sample. For the $10000 \mathrm{~V}$ ions, QE values were averaged only in the region where the ions were focused, as indicated by the red circle shown in Fig. 7 (right).

To determine if a specific surface cleave plane provided enhanced ion channeling, the QE results from the $10000 \mathrm{~V}$ ion implantation studies were divided by the nonimplantation QE results (Fig. 9). Per our supposition, when ions penetrate deeper into the material, they pass beyond the surface region that contributes to photoemission and therefore provide less $\mathrm{QE}$ degradation. When comparing $\mathrm{QE}$ values of implanted and nonimplanted samples, those with the highest QE ratio would correspond to samples with enhanced ion channeling. The simple picture presented in Fig. 2 suggests samples would be ordered (110), (111A) and (100), in terms of samples supporting the highest level of channeling to the least amount of channeling. This simple picture is consistent with results presented in Fig. 9, at least for samples that were heated sufficiently long and at a relatively high temperature (see results for heat cycles 4 and 5 in Fig. 9). The (110) GaAs cleave plane-i.e., the sample with the most open space between atoms, and therefore the sample supporting the highest level of ion channeling-exhibited the highest QE ratio. Whereas the cleave plane (100)-i.e., the sample with the least open 
space between atoms, and therefore the sample supporting the lowest level of ion channeling-exhibited the lowest $\mathrm{QE}$ ratio. If one assumes a strict $\mathrm{QE}$ dependence on atomic number density, the QE of (110) and (111A) cleave planes following ion implantation should be greater than sample (100) by roughly $41 \%$ and $15 \%$, respectively. In comparison, looking at Fig. 9 heat cycle 5 values, the measured QE of (110) and (111A) cleave planes following ion implantation are greater than sample (100) by $33 \%$ and $30 \%$, respectively, however there is fairly good agreement between measurements and predictions when error bars are taken into account.

\section{DISCUSSION}

We have shown that commercial bulk GaAs can be used to manufacture photocathodes with high QE without implementing complicated chemical surface treatments. Sample heating to $\sim 550^{\circ} \mathrm{C}$ inside a vacuum apparatus constructed using modern ultrahigh vacuum techniques was sufficient to obtain QE near $20 \%$ at $532 \mathrm{~nm}$ for all of the tested cleave plane surfaces, with cleave plane sample (100) providing the highest QE. For accelerator applications requiring high average current, photocathodes like these could produce more than $100 \mathrm{~mA}$ average current with just one Watt of laser power.

Implantation of the photocathode with either low or high energy hydrogen ions served to reduce photocathode QE, but the QE degradation caused by $100 \mathrm{~V}$ hydrogen ions was modest and QE could easily be recovered following sample heating. In sharp contrast, the QE degradation caused by $10000 \mathrm{~V}$ hydrogen ions was severe and QE could not be fully recovered with heat. These observations suggest that low energy ions penetrate the surface, stopping at locations between atoms and creating interstitial defects that likely reduce the diffusion length of the electrons within the material, reducing the number of available electrons that reach the photocathode surface that can contribute to photoemission. During sample heating, the interstitial defects diffuse throughout the material, restoring the electron diffusion length and the photocathode QE, whereas high energy ions likely generate knock-out vacancies within the crystal structure that permanently adversely alter the band structure of the semiconductor. As noted above, inside a dc high voltage photogun, the photocathode will be bombarded by considerably more low voltage ions than high voltage ions. This is fortuitous and explains why the photocathode QE can be routinely restored following simple heating and reactivation, except at the electrostatic center of the photocathode, where the highest energy ions are directed [8,27].

Our data support the simple picture that channeling is enhanced for the sample with the most open space between atoms. More open space leads to enhanced channeling which means high energy ions create fewer vacancies that lead to reduced and unrecoverable QE. Surface cleave plane (110) has the most open space and cleave plane (100) the least open space. As such, surface cleave plane (110) was the least sensitive to ion bombardment and cleave plane (100) the most sensitive. Considering all the results in total, the best choice for a photogun using bulk GaAs would be surface cleave plane (110), providing the highest $\mathrm{QE}$ and least sensitivity to ion bombardment. It is true that modern high-current accelerator applications now prefer alkali-antimonide photocathodes over GaAs photocathodes, because alkali-antimonide photocathodes offer similarly high QE but with longer operating lifetime. However, spin-polarized accelerator applications may be forced to use bulk GaAs as the only practical means to generate milliampere levels of spin-polarized beam [28].

We admit that the hydrogen ion source used in this experiment was rather primitive. Ion energy was set by the voltage applied to the sample during implantation, and not by the ion source itself, which introduced unappealing energy-dependent electrostatic focusing at the photocathode sample. It is important to note that ion implantation was performed with the photocathode NEA layer removed. This was done for a practical reason, namely, the ion source also emitted neutral atomic hydrogen which is a very effective chemical cleaning agent. The NEA layer was removed prior to implantation so as not to confuse $\mathrm{QE}$ reduction due to implantation with uninteresting $\mathrm{QE}$ reduction due to chemical removal of the $\mathrm{Cs}$ and $\mathrm{F}$ layer via atomic hydrogen cleaning. As such, our work did not directly evaluate photocathode sensitivity to sputtering of the Cs and F layer. However, because the ion dose required in this work was orders of magnitude greater than the ion dose delivered to the photocathode inside a photogun operating at $10^{-11}$ Torr, these results suggest ion sputtering of the NEA layer might be more deleterious than implantation. Future tests aimed at studying ion back-bombardment would benefit from using activated and unactivated photocathodes, and a dedicated ion source offering better control of the applied dose, the ion energy, and the ion distribution at the sample.

\section{ACKNOWLEDGMENTS}

Authored by Jefferson Science Associates, LLC under U.S. DOE Contract No. DE-AC05-06OR23177. The U.S. Government retains a non-exclusive, paid-up, irrevocable, world-wide license to publish or reproduce this manuscript for U.S. Government purposes.

[1] See, for example, Proceedings of the 21st International Symposium on Spin Physics (SPIN 2014), Beijing, China, 2014, edited by H. Gao and B.-Q. Ma [Int. J. Mod. Phys. Conf. Ser. 40, 1602001 (2016)].

[2] M. Kuwahara, S. Kusunoki, Y. Nambo, K. Saitoh, X. G. Jin, T. Ujihara, H. Asano, Y. Takeda, and N. Tanaka, Coherence of a spin-polarized electron beam emitted from a semiconductor photocathode in a transmission electron microscope, Appl. Phys. Lett. 105, 193101 (2014). 
[3] C. K. Sinclair, A $500 \mathrm{kV}$ photoemission electron gun for the CEBAF FEL, Nucl. Instrum. Methods Phys. Res., Sect. A 318, 410 (1992).

[4] P. A. Adderley, J. Clark, J. Grames, J. Hansknecht, K. Surles-Law, D. Machie, M. Poelker, M. L. Stutzman, and R. Suleiman, Load-locked dc high voltage GaAs photogun with an inverted-geometry ceramic insulator, Phys. Rev. ST Accel. Beams 13, 010101 (2010).

[5] E. Wang, D. Kayran et al., Fabrication of alkali antimonide photocathode for SRF gun, in Proceeding of IPAC 2014, Dresden, Germany (2014), p. 742, MOPRI 059, http:// accelconf.web.cern.ch/accelconf/IPAC2014/papers/ mopri059.pdf.

[6] B. Dunham, A. Bartnik et al., Record high-average current from a high-brightness photoinjector, Appl. Phys. Lett. 102, 034105 (2013).

[7] K. Aulenbacher et al., SLAC Report No. 432, 1993.

[8] J. Grames, R. Suleiman, P. A. Adderley, J. Clark, J. Hansknecht, D. Machie, M. Poelker, and M. L. Stutzman, Charge and fluence lifetime measurements of a dc high voltage GaAs photogun at high average current, Phys. Rev. ST Accel. Beams 14, 043501 (2011).

[9] K. A. Elamrawi and H. E. Elsayed-Ali, GaAs photocathode cleaning by atomic hydrogen from a plasma source, J. Phys. D 32, 251 (1999).

[10] M. Baylac, P. Adderley, J. Brittian, J. Clark, T. Day, J. Grames, J. Hansknecht, M. Poelker, M. Stutzman, A. T. $\mathrm{Wu}$, and A. S. Terekhov, Effects of atomic hydrogen and deuterium exposure on high polarization GaAs photocathodes, Phys. Rev. ST Accel. Beams 8, 123501 (2005).

[11] M. T. Robinson and O. S. Oen, The channeling of energetic atoms in crystal lattices, Appl. Phys. Lett. 2, 30 (1963).

[12] K. Nordlund, Molecular dynamics simulation of ion ranges in the 1-100 keV energy range, Comput. Mater. Sci. 3, 448 (1995).

[13] M. Reiser, Theory and Design of Charged Particle Beams (John Wiley and Sons, Inc., New York, 1994).

[14] J. J. Scheer and J. van Laar, GaAs-Cs: A new type of photoemitter, Solid State Commun. 3, 189 (1965).
[15] A. A. Turnbull and G. B. Evans, Photoemission from GaAs-Cs-O, J. Phys. D: Appl. Phys. 1, 155 (1968).

[16] L. W. James, G. A. Antypas, J. Edgecumbe, R. L. Moon, and R. L. Bell, Dependence on crystalline face of the band bending in $\mathrm{Cs} 2 \mathrm{O}$ activated GaAs, J. Appl. Phys. 42, 4976 (1971).

[17] R. W. Hamm and M. E. Hamm, Industrial Accelerators and Their Applications (World Scientific, Singapore, 2012).

[18] J. Biersack, J. P. Ziegler, and M. D. Ziegler, http://www .srim.org.

[19] E. D. Palik, Handbook of Optical Constants of Solids Volume 1 (Academic Press, Orlando, 1985), p. 438.

[20] M. D. Sturge, Optical absorption of gallium arsenide between 0.6 and $2.75 \mathrm{eV}$, Phys. Rev. 127, 768 (1962).

[21] S. Zollner and D. Zarr, Optical constants of GaAs from 0.73 to $6.60 \mathrm{eV}$ for dielectric film thickness metrology in compound semiconductor manufacturing, in IEEE International Symposium on Compound Semiconductor (2000), pp. 13-18.

[22] L. W. Aukerman, M. F. Millea, and M. McColl, Diffusion lengths of electrons and holes in GaAs, J. Appl. Phys. 38, 685 (1967).

[23] J. Wilms and W.E. Spicer, Quantum efficiency and radiative lifetime in p-type gallium arsenide, J. Appl. Phys. 36, 2815 (1965).

[24] W. W. MacAlpine and R. O. Schildknecht, Coaxial resonators with helical inner conductor, Proc. IRE 47, 2099 (1959).

[25] CST Studio Suite: https://www.cst.com.

[26] K. L. Jensen, D. W. Feldman, N. A. Moody, and P. G. O'Shea, A photoemission model for low work function coated meatal surfaces and its experimental validation, J. Appl. Phys. 99, 124905 (2006).

[27] C. K. Sinclair, P. A. Adderley, B. M. Dunham, J. C. Hansknecht, P. Hartmann, M. Poelker, J. S. Price, P. M. Rutt, W. J. Schneider, and M. Steigerwald, High average current polarized electron source with long cathode operational lifetime, Phys. Rev. ST Accel. Beams 10, 023501 (2007).

[28] eRHIC zeroth order design report, edited by $\mathbf{M}$. Farkhondeh and V. Ptitsyn, https://www.bnl.gov/cad/ eRhic/eRHIC_ZDR.asp. 